\title{
Evolution of gravitational waves through the cosmological QCD transition
}

\author{
Dominik J. Schwarz \\ Institut für Theoretische Physik, Universität Frankfurt, \\ Postfach 1119 32, 60054 Frankfurt am Main, Germany*
}

11 August 1998

\begin{abstract}
The spectrum of gravitational waves that have been produced in inflation is modified during cosmological transitions. Large drops in the number of relativistic particles, like during the QCD transition or at $e^{+} e^{-}$annihilation, lead to steps in the spectrum of gravitational waves. We calculate the transfer function for the differential energy density of gravitational waves for a first-order and for a crossover QCD transition.
\end{abstract}

\section{Introduction}

Detecting a stochastic background of gravitational waves [1] would open a new window to the early Universe. Primordial gravitational waves are predicted to be generated during inflation [2] and could be detected with upcoming cosmic microwave background observations. Defects, like cosmic strings, produce stochastic gravitational waves as well [3]. Inflation and defects predict an almost scaleinvariant energy density per logarithmic frequency interval for the most interesting frequencies $\left(\sim 10^{-8} \mathrm{~Hz}\right.$ for pulsar timing, $\sim 10^{-3} \mathrm{~Hz}$ for LISA, and $\sim 100 \mathrm{~Hz}$ for LIGO) of gravitational waves.

The aim of this paper is to study the evolution of primordial gravitational waves through transitions of the equation of state, especially the QCD transition. A step in the gravitational wave spectrum comes from the large drop of the number of relativistic particles (by a factor $\sim 3$ ) during the QCD transition. Since entropy is conserved, the growth rate of the Hubble radius $H^{-1}$ is diminished during the transition. Thus, the rate at which modes cross into the horizon is changed during the transition and a step in the spectrum shows up at

*e-mail: dschwarz@th.physik.uni-frankfurt.de 
frequencies of order of the Hubble rate at the transition. For the QCD transition this frequency is $10^{-7} \mathrm{~Hz}$ today and the step is a $30 \%$ correction. Other large drops in entropy density happen at $e^{+} e^{-}$annihilation (which gives rise to a $20 \%$ correction in the energy spectrum) and at a GUT phase transition. The typical frequencies are $10^{-10} \mathrm{~Hz}$ for $e^{+} e^{-}$annihilation and $10^{9} \mathrm{~Hz}$ for the GUT transition.

Similar steps in the differential spectrum have been studied for gravitational waves generated by cosmic strings [3]. These gravitational waves are generated on subhorizon scales when the cosmic strings decay. Their frequency is $>\alpha^{-1} H$, where $\alpha$ is the ratio between the typical size of a string loop and the Hubble radius at formation of the loop. $\alpha$ is smaller than 0.1 and might be as small as $10^{-5}$ [4]. In this situation steps in the differential spectrum follow from the conservation of entropy for decoupled species (gravitons) during cosmological phase transitions [5]. This interpretation applies to modes that have been inside the horizon long before the transition or that have been generated on subhorizon scales. However, for superhorizon modes entropy and energy of a gravitational wave are not defined. We therefore cannot rely on the conservation of entropy argument when dealing with gravitational waves from inflation.

In Sec. 2 we briefly recapitulate the generation of primordial gravitational waves from inflation. The equation of state during the QCD transition is discussed in Sec. 3. In Sec. 4 we explain the origin of the step in the spectrum and present results from numerical calculations, for a first order QCD transition and for a QCD crossover. A further discussion of the steps in the spectrum of primordial gravitational waves from the QCD transition and other cosmological transitions, especially $e^{+} e^{-}$annihilation, is presented in Sec. 5.

\section{Primordial spectrum of gravitational waves}

Let us recall the production of gravitational waves during inflation [2, 6]. The line element of gravitational waves is given by $\mathrm{d} s^{2}=-\mathrm{d} t^{2}+a^{2}\left(\delta_{i j}+h_{i j}\right) \mathrm{d} x^{i} \mathrm{~d} x^{j} . h_{i j}$ is a transverse, traceless tensor. $a$ denotes the scale factor. The spatial average $\left\langle h_{i j}(x) h^{i j}(x+r)\right\rangle=\int j_{0}(k r) k^{3}\left|h_{k}\right|^{2} \mathrm{~d} \ln k$ defines the power spectrum $\left|h_{k}\right|^{2} . k$ is the comoving wavenumber. We define the rms amplitude $h$ of a gravitational wave per logarithmic frequency interval: $h \equiv k^{3 / 2}\left|h_{k}\right|$. The linearized equation of motion for $h(t)$ reads

$$
\ddot{h}+3 H \dot{h}+\frac{k^{2}}{a^{2}} h=0,
$$

where the differentiation is taken with respect to cosmic time $t$ and $H \equiv \dot{a} / a$.

During the quasi-de Sitter period gravitational waves are produced with almost scale-invariant spectrum. For superhorizon scales, $k_{\mathrm{ph}} \equiv k / a \ll H$, the slow roll approximation gives [6]:

$$
h^{2}=\frac{16}{\pi} F(\epsilon)\left(\frac{H_{\mathrm{dS}}}{M_{\mathrm{P}}}\right)_{k=H a}^{2},
$$


where $\epsilon \equiv-\dot{H} / H^{2} \ll 1$ is the slow roll parameter and $F(\epsilon)=1-\left(\gamma_{\mathrm{E}}+\ln 2-\right.$ $1) \epsilon+\mathcal{O}\left(\epsilon^{2}\right) ; \gamma_{\mathrm{E}}+\ln 2-1 \approx 0.27$. The amplitude of gravitational waves stays constant until the second horizon crossing and decays as $1 / a$ thereafter, $h \simeq$ $C_{k} \sin \left(k \eta+\delta_{k}\right) / a$, where $\eta=\int \mathrm{d} t / a$ is conformal time. $C_{k}$ and $\delta_{k}$ are determined by matching to the superhorizon solution.

For subhorizon modes, $k_{\mathrm{ph}} \gg H$, the energy density of gravitational waves can be defined. The space-time average of the energy-momentum tensor over several wavelengths gives $\rho_{\mathrm{g}}=-\left(M_{\mathrm{P}}^{2} / 32 \pi\right)\left\langle\dot{h}_{i j} \dot{h}^{i j}\right\rangle$. The energy density per logarithmic interval in $k$ is related to the rms amplitude $h$ :

$$
k \frac{\mathrm{d} \rho_{\mathrm{g}}}{\mathrm{d} k}=\frac{M_{\mathrm{P}}^{2}}{32 \pi} k_{\mathrm{ph}}^{2} \frac{1}{2} h^{2} .
$$

The factor $1 / 2$ comes from the time average over several oscillations. The energy fraction in gravitational waves, per logarithmic interval in $k$, is defined by

$$
\Omega_{\mathrm{g}}(k) \equiv k \frac{\mathrm{d} \rho_{\mathrm{g}}}{\mathrm{d} k} \frac{1}{\rho_{\mathrm{c}}},
$$

where $\rho_{c} \equiv 3 M_{\mathrm{P}}^{2} H_{0}^{2} /(8 \pi)$. Finally, the fractional energy density per logarithmic frequency interval reads:

$$
\Omega_{\mathrm{g}}(k) \simeq \frac{2}{3 \pi} F(\epsilon)\left(\frac{H_{\mathrm{dS}}}{M_{\mathrm{P}}}\right)^{2}\left(\frac{k_{\mathrm{ph}}}{H_{0}}\right)^{2}\left(\frac{a_{\mathrm{hc}}}{a_{0}}\right)^{2} .
$$

By $\simeq$ we indicate that we replaced the exact time dependence of $h$ by $a_{\mathrm{hc}} / a_{0}$. Modes that cross into the horizon $\mathbb{P}$ in the matter dominated epoch have $a_{\mathrm{hc}} / a_{0}=$ $\left(H_{0} / k_{\mathrm{ph}}\right)^{2}$ and modes that cross into the horizon in the radiation dominated regime have $a_{\mathrm{hc}} / a_{0}=H_{0} /\left[\left(1+z_{\mathrm{eq}}\right)^{1 / 2} k_{\mathrm{ph}}\right]$. From Eq. (5) we obtain

$$
\Omega_{\mathrm{g}}(k) \simeq \frac{2}{3 \pi} F(\epsilon)\left(\frac{H_{\mathrm{dS}}}{M_{\mathrm{P}}}\right)^{2}\left\{\begin{array}{ll}
\left(H_{0} / k_{\mathrm{ph}}\right)^{2} & H_{0}<k_{\mathrm{ph}}<H_{0}\left(1+z_{\mathrm{eq}}\right)^{1 / 2} \\
\left(1+z_{\mathrm{eq}}\right)^{-1} & H_{0}\left(1+z_{\mathrm{eq}}\right)^{1 / 2}<k_{\mathrm{ph}}<H_{d S}
\end{array} .\right.
$$

For comparison with experimental limits we use the frequency today, $f \equiv$ $2 \pi k_{\mathrm{ph}}\left(t_{0}\right)$. In inflationary cosmology the strongest limit on $\Omega_{\mathrm{g}}$ comes from the anisotropies of the cosmic microwave background []]. This limit yields $\Omega_{\mathrm{g}} h_{50}^{2}<$ $3 \times 10^{-10}\left(H_{0} / f\right)^{2}$ in the frequency range $H_{0}<f<30 H_{0}$ [8, 9]. From Eq. (6) we have $\Omega_{\mathrm{g}} h_{50}^{2}<7 \times 10^{-12} / z_{\mathrm{eq}} \approx 1 \times 10^{-15}$ for modes that crossed into the horizon during the radiation dominated epoch. Direct limits on $\Omega_{\mathrm{g}} h_{50}^{2}$ in the radiation era have been obtained by pulsar timing $\left(<4 \times 10^{-7}\right.$ for $f \approx 4 \times 10^{-9}$ to $\left.4 \times 10^{-8} \mathrm{~Hz}\right) 10$, 11 and from big bang nucleosynthesis $\left(\int \Omega_{\mathrm{g}} \mathrm{d}(\ln f)<10^{-5}\right.$ for $f>H_{\mathrm{BBN}} \sim 10^{-9} \mathrm{~Hz}$ ) [12]. Although, inflation predicts an extremely small amount of energy in gravitational waves at small scales, this is different for other

\footnotetext{
${ }^{1}$ We define the moment of horizon crossing by the condition $k_{\mathrm{ph}}=H$.
} 
models of structure formation. Defects might generate a scale-invariant spectrum of gravitational waves with amplitudes that may be seen in pulsar timing residuals in the near future. String cosmology [13] predicts a different energy spectrum, $\propto$ $f^{3}$, thus the BBN constraint, which does not constrain the inflationary scenarios at all, turns into a severe restriction.

\section{The cosmological QCD transition}

Lattice QCD results indicate that QCD makes a transition from a phase of free quarks and gluons to the phase of hadrons at a temperature $T_{\star} \approx 150 \mathrm{MeV}$ [14]. This implies a Hubble radius $R_{\mathrm{H}} \sim 10 \mathrm{~km}$ at the transition. Modes that crossed the horizon during the QCD transition have frequencies $f_{\star} \sim 10^{-7} \mathrm{~Hz}$ today. The order of the QCD transition is still a subject of debate. Lattice QCD with Wilson quarks indicates that the QCD transition is of first order for the physical values of the quark masses [15], whereas results with staggered quarks [16] indicate a crossover for the physical quark masses.

We consider both scenarios for the cosmological QCD transition, a first-order phase transition and a smooth crossover. A first-order QCD transition starts with a short period of supercooling and the nucleation of hadron bubbles. The supercooling is tiny, $\delta T / T \sim 10^{-3}$, which implies that the entropy production is negligible, $\Delta S / S \sim 10^{-6}[18,19]$. After enough hadron bubbles have been nucleated to reheat the Universe to the critical temperature, further bubble formation is suppressed and the bubbles grow adiabatically during the remaining 99\% of the transition. For our purpose the cosmic fluid remains in thermal equilibrium throughout the phase transition. From recent lattice QCD results [17] for the surface tension and for latent heat we find that the typical bubble separation is $\sim 10^{-6} R_{\mathrm{H}} 19$.

A simple model for a first-order transition is given by the bag model [20]. In this model the entropy density jumps at the critical temperature $T_{\star}$

$$
s(T)=\frac{2 \pi^{2}}{45} g_{\mathrm{a}} T^{3}\left[1+\frac{\Delta g}{g_{\mathrm{a}}} \theta\left(T-T_{\star}\right)\right],
$$

where the effective number of relativistic helicity degrees of freedom before the transition are $g_{\mathrm{b}}=51.25$ (2 quark flavors, photons, and leptons 4 ) and $g_{\mathrm{a}}=17.25$ (pions, photons, and leptons) after the transition; $\Delta g \equiv g_{\mathrm{b}}-g_{\mathrm{a}}$. At $T_{\star}$ the high and low temperature phases can coexist.

For a smooth crossover we assume for the entropy density [21]

$$
s(T)=\frac{2 \pi^{2}}{45} g_{\mathrm{a}} T^{3}\left[1+\frac{1}{2} \frac{\Delta g}{g_{\mathrm{a}}}\left[1+\tanh \left(\frac{T-T_{\star}}{\Delta T}\right)\right]\right] .
$$

\footnotetext{
${ }^{2} e^{ \pm}, \mu^{ \pm}$, and all $\nu \mathrm{s}$.
} 
We recover the bag model for $\Delta T \rightarrow 0$. Both models coincide at temperatures far away from $T_{\star}$.

In both models the pressure is determined from $p=\int s \mathrm{~d} T$ and the energy density follows from the second law of thermodynamics, $\rho=s T-p$. The relation between the temperature and the scale factor follows from the conservation of entropy,

$$
\frac{\mathrm{d} T}{\mathrm{~d} \ln a}=-\frac{3 s}{\mathrm{~d} s / \mathrm{d} T},
$$

except for $T=T_{\star}$ in the case of a first-order phase transition. In the bag model $T \propto 1 / a$ for $T \neq T_{\star}$. During a first-order QCD transition, i.e. $T=T_{\star}$, the pressure $p\left(T_{\star}\right) \equiv p_{\star}$ is constant. The energy density $\rho(a)$ is obtained from the first law of thermodynamics $d \rho=-3\left(\rho+p_{\star}\right) d a / a$. In the bag model the transition lasts a third of a Hubble time. For a detailed discussion of the cosmological QCD transition see Ref. [19].

\section{Step in the differential energy spectrum}

Figure 1 shows the transfer function $\Omega_{\mathrm{g}}(f) / \Omega_{\mathrm{g}}\left(f \ll f_{\star}\right)$ from a numerical integration of Eq. (1). The typical frequency scale is

$$
f_{\star} \approx 1.36\left(\frac{g}{17.25}\right)^{\frac{1}{2}} \frac{T_{\star}}{150 \mathrm{MeV}} 10^{-7} \mathrm{~Hz},
$$

which corresponds to the mode that crosses the Hubble horizon at the end of the bag model QCD transition. Scales that cross into the horizon after the transition (l.h.s. of the figure) are unaffected, whereas modes that cross the horizon before the transition are damped by an additional factor $\approx 0.7$. The modification of the differential spectrum has been calculated for a first-order QCD transition (bag model), Eq. (7), and a crossover QCD transition, Eq. (8), with $\Delta T / T_{\star}=0.3$. In both cases the step extends over one decade in frequency. The detailed form of the step is almost independent from the order of the transition. To solve Eq. (1) numerically, it proofs useful to use the scale factor as evolution parameter for the first order transition and the temperature for the crossover transition. $a$ and $T$ are related by Eq. (9).

The size of the step can be calculated analytically: Long before and after the transition, in the radiation dominated era,

$$
H \propto g^{-1 / 6} a^{-2},
$$

from the Friedmann equation and the conservation of entropy. On the other hand, $k_{\mathrm{ph}} \propto 1 / a$, independently of the equation of state. Thus, the rate at which modes enter the Hubble horizon is changing during the transition. This change leads to the step in the transfer function. 


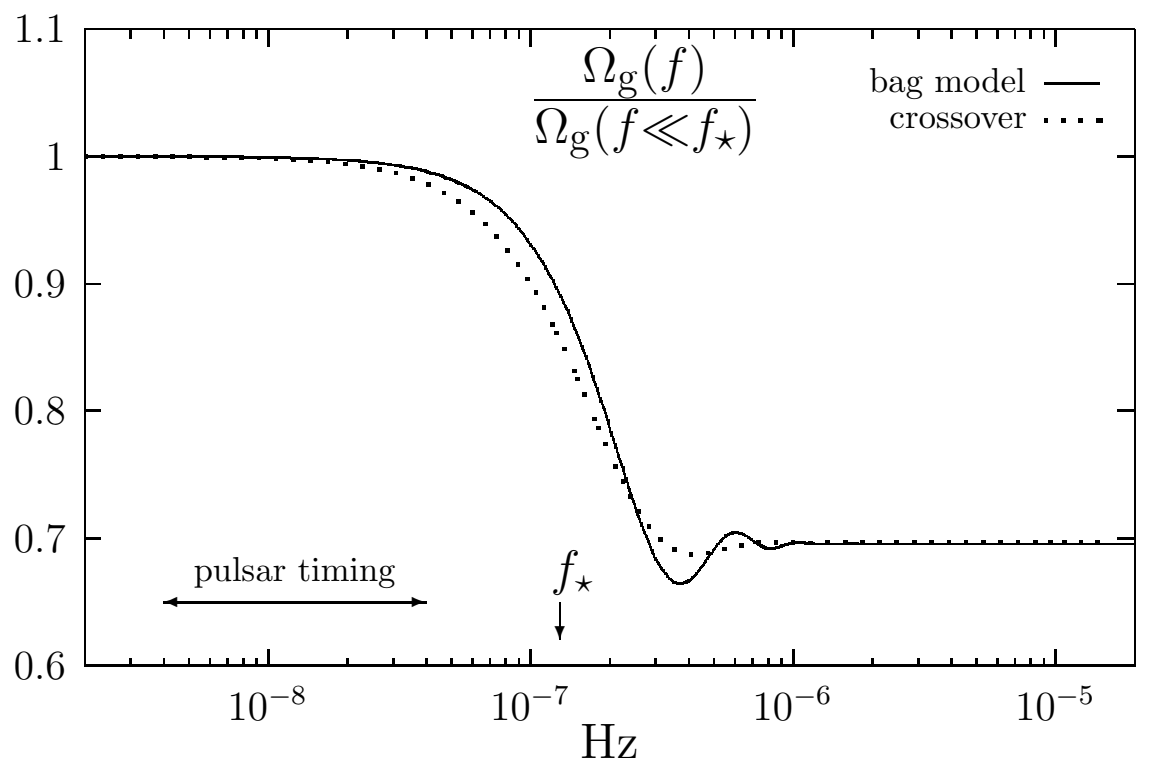

Figure 1: The modification of the energy density, per logarithmic frequency interval, for primordial gravitational waves from the QCD transition.

For a fixed mode $k_{\mathrm{a}}$ that crosses the horizon after the phase transition the amplitude $h$ is constant during the transition, the transfer function is one. Now, consider a mode $k_{\mathrm{b}}$ that crosses the horizon before the transition. Its amplitude decays proportionally to $a_{\mathrm{hc}} / a$. From Eq. (5) we find

$$
\Omega_{\mathrm{g}}\left(k_{\mathrm{b}}\right) \propto k_{\mathrm{ph}}^{2} a_{\mathrm{hc}}^{2} \propto g_{\mathrm{b}}^{-1 / 3},
$$

where we have used Eq. (11) and $k_{\mathrm{ph}}=H$ at horizon crossing. The constants of proportionality have been neglected in Eq. (12), because they drop out from the transfer function below. Comparing the differential energy spectrum for modes that cross into the horizon before the transition and for modes that cross into the horizon after the transition gives the ratio

$$
\frac{\Omega_{\mathrm{g}}\left(f \gg f_{\star}\right)}{\Omega_{\mathrm{g}}\left(f \ll f_{\star}\right)}=\left(\frac{g_{\mathrm{a}}}{g_{\mathrm{b}}}\right)^{\frac{1}{3}} \approx 0.696,
$$

for the QCD transition, which coincides with the numerical integration in Fig. 1. The size and position of the step in the logarithmic spectrum is independent of the order of the transition!

The result (13) is in agreement with the entropy conservation of subhorizon gravitational waves. However, for superhorizon modes the entropy is not defined.

Let us estimate the slope of the step in the transfer function. From the Friedmann equation and the 1st law of thermodynamics we obtain the Hubble 
rate as a function of the scale factor $(w \equiv p / \rho)$

$$
\mathrm{d} \ln H(a)=-\frac{3}{2}[1+w(a)] \mathrm{d} \ln a .
$$

For $0<w \leq 1 / 3$ during the phase transition the Hubble expansion changes during the transition, i.e., $H \propto a^{-\beta}$ with $\beta=\beta(a)$ in the range $(3 / 2,2)$. This holds true if the change in $w$ is small, i.e. $(\mathrm{d} w / \mathrm{d} a) \Delta a \ll 1+w$. To estimate the exponent

$$
n(f) \equiv \frac{\mathrm{d} \ln \Omega_{\mathrm{g}}(f)}{\mathrm{d} \ln f}
$$

we use the relation $\mathrm{d} \ln f=\mathrm{d} \ln (H a)_{\mathrm{hc}}=[-(1+3 w) / 2 \mathrm{~d} \ln a]_{\mathrm{hc}}$ and Eq. (5). We find

$$
n(f) \simeq-2 \frac{1-3 w\left(a_{\mathrm{hc}}\right)}{1+3 w\left(a_{\mathrm{hc}}\right)}
$$

which takes values between -2 and 0 for $w$ between 0 and $1 / 3$. From the numerical integration (see Fig. 1) we find that the minimum of the exponent is $n \approx-0.8$ for the bag model, which corresponds to an effective value $w_{\text {eff }} \approx 0.15$. This is consistent with the values $w(a) \in(0.09,1 / 3)$ that are taken during the first-order QCD transition. For the crossover transition the drop in $w$ is smaller and thus the step is not as steep as in the first-order transition.

\section{Discussion}

In Fig. 1 we indicated the frequency range $\left(\sim 1 \mathrm{yr}^{-1}\right)$ in which limits on $\Omega_{\mathrm{g}}$ have been reported from pulsar timing residuals [11]. The frequencies where the step of the QCD transition would be visible is of the order $0.3 \mathrm{month}^{-1}$. For pulsar timing the power spectrum of gravitational waves is more relevant than the energy spectrum. The power spectrum is $\propto \Omega_{\mathrm{g}}(f) f^{-5}$. Our results show that the power spectrum might deviate from the $f^{-5}$ behavior over a whole decade in frequency. Depending on the effective value of $w$ during the transition it might be as steep as $f^{-7}$ (for $w=0$ ). However, a realistic estimate for the QCD transition gives $w_{\text {min }} \geq 0.1$. Thus, the spectral index of the power spectrum, $n-4$, lies, according to Eq. (16), between -6.1 and -5 .

The $30 \%$ step in the differential spectrum from the QCD transition might be bigger than the tilt of the spectrum of gravitational waves from inflation (see [9] for a computation of the tilt for various inflationary models), e.g. in chaotic inflation.

As mentioned in the introduction, similar steps in the spectrum might occur at $e^{+} e^{-}$annihilation, where $f_{\text {ann }} \sim 10^{-10} \mathrm{~Hz}$ is the typical frequency. Eq. (13) is modified for $e^{+} e^{-}$annihilation, because neutrinos and photons are decoupled. 
Taking their difference in temperature into account $\left[T_{\nu} / T_{\gamma}=(4 / 11)^{1 / 3}\right]$ we obtain

$$
\frac{\Omega_{\mathrm{g}}\left(f \gg f_{\mathrm{ann}}\right)}{\Omega_{\mathrm{g}}\left(f \ll f_{\mathrm{ann}}\right)}=\frac{\left[g_{\gamma}+\frac{4}{11} g_{\nu}\right]^{\frac{4}{3}}\left[g_{\gamma}+\left(\frac{4}{11}\right)^{\frac{4}{3}} g_{\nu}\right]^{-1}}{g_{\mathrm{b}}^{\frac{1}{3}}} \approx 0.8
$$

where $g_{\gamma}=2, g_{\nu}=5.25$, and $g_{b}=10.75$. Again, this result is in agreement with the entropy conservation of subhorizon gravitational waves.

For the electroweak transition the typical frequency $\left(\sim 6 \times 10^{-4} \mathrm{~Hz}\right)$ lies in the frequency range of LISA, however the modification of the spectrum is tiny (the only particle that disappears from the radiation fluid is the Higgs particle).

We have studied the evolution of gravitational waves through the QCD transition in some detail. Although the detection of primordial gravitational waves is unlikely in the near future, it is exciting that the particle content in the early Universe is remembered in the spectrum of gravitational waves today. The spectrum contains information about the relativistic degrees of freedom at a certain epoch of the early Universe and the transition temperatures of various cosmological transitions. On the other hand, if we assume to know the QCD transition temperature from lattice QCD, measuring the position of the step in the differential energy spectrum would tell us whether the gravitational waves have been created during inflation or in a more recent epoch of the Universe $\left(f_{\star} \sim 10^{-7} \mathrm{~Hz}\right.$ from inflation, $>10^{-5} \mathrm{~Hz}$ from cosmic strings).

\section{Acknowledgments}

I thank P. Widerin for helping me with the numerical calculations and R. Caldwell, V. Mukhanov, C. Schmid, and P. Widerin for comments and discussions. This work has been supported by the Swiss National Science Foundation and by the Alexander von Humboldt foundation.

\section{References}

[1] Reviews on the generation and detection of stochastic gravitational waves can be found in: K. S. Thorne, in 300 Years of Gravitation (eds. S. Hawking and W. Israel, Cambridge University Press, Cambridge, 1987) pp. 330; B. Allen, Les Houches Lecture Notes Sept. 1995, preprint gr-qc/9604033.

[2] A. A. Starobinskii, Pis'ma Zh. Eksp. Teor. Fiz. 30, 719 (1979) [JETP Lett. 30, 682 (1979)]; L. F. Abbott and D. D. Harrari, Nucl. Phys. B 264, 487 (1986); B. Allen, Phys. Rev. D 37, 2078 (1988); V. F. Mukhanov, H. A. Feldman, and R. H. Brandenberger, Phys. Rep. 215, 203 (1992). 
[3] A. Vilenkin, Phys. Lett. 107B, 47 (1981); A. Vilenkin and E. P. S. Shellard, Cosmic Strings and Other Topological Defects (Cambridge University Press, Cambridge, 1994) pp. 306.

[4] R. R. Caldwell and B. Allen, Phys. Rev. D 45, 3447 (1992).

[5] D. P. Bennett, Phys. Rev. D 34, 3592 (1986); Phys. Rev. D 34, 3932 (1986). R. R. Caldwell, R. A. Battye, and E. P. S. Shellard, Phys. Rev. D 54, 7146 (1996).

[6] E. D. Stewart and D. H. Lyth, Phys. Lett. B 302, 171 (1993).

[7] C. L. Bennett et al., Astrophys. J. 464, L1 (1996).

[8] V. A. Rubakov, M. V. Sazhin and A. V. Veryaskin, Phys. Lett. 115B, 189 (1982); R. Fabbri and M. D. Pollock, ibid. 125B, 445 (1983); L. F. Abbott and M. B. Wise, Nucl. Phys. B 244, 541 (1984); A. A. Starobinskii, Pis'ma Astron. Zh. 11, 323 (1985) [Sov. Astron. Lett. 11, 133 (1985)]; M. S. Turner, M. White, and J. E. Lidsey, Phys. Rev. D 48, 4613 (1993).

[9] M. S. Turner, Phys. Rev. D 48, 3502 (1993).

[10] M. V. Sazhin, Astron. Zh. 55, 65 (1978) [Sov. Astron. 22, 36 (1978)]; S. Detweiler, Astrophys. J. 234, 1100 (1979).

[11] V. M. Kaspi, J. H. Taylor, and M. F. Ryba, Astrophys. J. 428, 713 (1994); S. E. Thorsett and R. J. Dewey, Phys. Rev. D 53, 3468 (1996); M. P. McHugh et al., Phys. Rev. D 54, 5993 (1996).

[12] B. J. Carr, Astron. Astrophys. 86, 6 (1980).

[13] G. Veneziano, Helv. Phys. Acta 69, 553 (1996) and references therein.

[14] For recent reviews see K. Kanaya, Nucl. Phys. B (Proc. Suppl.) 47, 144 (1996); E. Laermann, Nucl. Phys. B (Proc. Suppl.) 63, 141 (1998).

[15] Y. Iwasaki et al., Z. Phys. C 71, 343 (1996).

[16] F. R. Brown et al., Phys. Rev. Lett. 20, 2491 (1990).

[17] Y. Iwasaki et al., Phys. Rev. D 46, 4657 (1992); 49, 3540 (1994); B. Beinlich, F. Karsch, and A. Peikert, Phys. Lett. B 390, 268 (1997).

[18] E. Witten, Phys. Rev. D 30, 272 (1984); J. Ignatius et al., Phys. Rev. D 49, 3854 (1994); 50, 3738 (1994); M. B. Christiansen and J. Madsen, Phys. Rev. D 53, 5446 (1996). 
[19] C. Schmid, D. J. Schwarz, and P. Widerin, Phys. Rev. Lett. 78, 5468 (1997); astro-ph/9807257 (1998).

[20] T. DeGrand and K. Kajantie, Phys. Lett. 147B, 273 (1984).

[21] J. P. Blaizot and J. Y. Ollitrault, Phys. Rev. D 36, 916 (1987). 